\title{
Doppelspitzen: Notlösung, Heilsbringer oder innovatives Führungsmodell? Coaching für gelingende Führungstandems
}

\author{
Christiane Münderlein
}

Eingegangen: 1. November 2020 / Angenommen: 2. Februar 2021 / Online publiziert: 25. März 2021

(C) Der/die Autor(en) 2021

Zusammenfassung Doppelspitzen werden in der aktuellen Führungsdebatte kontrovers diskutiert. Wissenschaftliche Erkenntnisse zur Arbeit von Führungstandems sind rar. Bisherige Coachingkonzepte befassen sich schwerpunktmäßig mit dem Konfliktcoaching von Doppelspitzen. In dem vorliegenden Beitrag wird den Fragen nachgegangen, welche Gelingensfaktoren und Herausforderungen es bei geteilter Leitung gibt und wie die Potenziale von Führungstandems durch (präventives) Coaching gehoben werden können.

Schlüsselwörter Doppelspitzen · Topsharing · Coaching

\section{Dual Leadership: Emergency solution, savior or innovative management model? Coaching for successful leadership tandem}

\begin{abstract}
Dual leadership situations are being controversially talked about in current leadership debate. Scientific knowledge of the work of leadership tandem is rare. Previous coaching concepts have focused on conflict coaching for dual peaks. In this article, the questions of which success factors and challenges exist with shared management and how the potential of leadership tandem can be raised through (preventive) coaching is pursued.
\end{abstract}

Keywords Dual leadership · Topsharing · Coaching

C. Münderlein $(\triangle)$

Hallerstr. 44, 90419 Nürnberg, Deutschland

E-Mail: kontakt@christiane-muenderlein.de

Universität Kassel, Kassel, Deutschland 
Nach nur sechs Monaten scheitert Mitte April 2020 die Doppelspitze von Jennifer Morgan und Christian Klein im Vorstandsvorsitz des DAX-Unternehmens SAP. „Die Corona-Krise verlange ein entschlossenes Handeln und eine klare, hierbei unterstützende Führungsstruktur“, heißt es in einer Mitteilung von SAP (Nezik 2020, S. 20). „Morgan und Klein (...) verkörperten einen Aufbruch in eine neue Zeit, den endgültigen Abschied von der Deutschland AG, dem Zirkel der immer gleichen Herren, die über Jahrzehnte die Geschicke der wichtigsten deutschen Unternehmen lenkten und kontrollierten“ (ebd.). Auch gesellschaftlich scheint die Corona-Pandemie wieder die One-Man-Show in Politik und Unternehmen zu beflügeln.

Wissenschaftliche Erkenntnisse zur Arbeit von Doppelspitzen sind rar. Häufig werden sie in der Praxis als Notlösung, z. B. bei der Fusion von zwei Unternehmen oder beim Thema Familienarbeit und Karriere als Vereinbarkeitsthema verhandelt. Die Praxis ist aber voll von gelingenden und scheiternden Führungstandems. Erfolgreiche Gründerpaare, z. B. bei Google mit Larry Page und Sergey Brin, sind genauso vertreten wie geschichtsträchtige Konfliktkonstellationen der Dassler Brüder mit Adidas und Puma. In aktuellen Veröffentlichungen erscheint die Doppelspitze als Lösung für agile Unternehmen. Unter Schlagworten wie „Nimm 2 - moderne Führung und Agilität“, ,Doppel(t)spitze - zu zweit mehr Erfolg“, ,die Doppelspitze eine Chance für die Führungskultur?" (Hegewald 2017) klingen hauptsächlich positive Seiten dieser Führungskonstellation an. Die Doppelspitze von BÜNDNIS 90/Die GRÜNEN mit Annalena Baerbock und Robert Habeck ist derzeit äußerst erfolgreich. So stellen sich die Fragen, was die Herausforderungen und Gelingensfaktoren von Doppelspitzen sind, wo die Potenziale liegen und unter welchen Bedingungen ein erfolgreiches Arbeiten des Führungsduos möglich ist.

\section{Begründungen für geteilte Leitung}

Im Wesentlichen werden drei Begründungsstränge für die Besetzung von Doppelspitzen genannt: (1) Verteilung von Macht, (2) Teilzeitführung als attraktive PEMaßnahme, (3) Herausforderungen durch zunehmende Komplexität und Agilität.

Verteilung von Macht: Große, kaum beschränkte Macht in den Händen einzelner Führungspersonen an der Spitze von Staaten, Parteien und Organisationen führen nach vielfältigen Erkenntnissen immer wieder zu Machtmissbrauch. Die Ethikforschung verweist auf die Gefahr moralisch verwerflicher und korrupter Verhaltensweisen bei Machtkonzentration ,durch die weit verbreitete elitäre Abkoppelung von Führungsriegen sowie Bestrebungen, sich statusmäßig von anderen abzuheben“ (Endres und Weibler 2019, S. 26). Eine mögliche Antwort ist, Führung auf mehrere Personen zu verteilen.

Attraktive und gendersensible Führungsmodelle und Teilzeitführung als Personalentwicklungsmaßnahme: Als Begründung für Organisationen wird die höhere Arbeitgeber-Attraktiviät durch moderne Führungsmodelle genannt. Die Recruiting-Vorteile beziehen sich insbesondere auf gut ausgebildete Wissensarbeiter/ innen (ebd., S. 25). Arbeitnehmer/innen fordern zunehmend sinnhafte und zu ihren Lebensbezügen passende Arbeitsmodelle. Durch das Teilzeit- und Befristungsge- 
setz gibt es grundsätzlich auch eine Verpflichtung des Arbeitgebers, Führung in Teilzeit anzubieten. Teilzeitmodelle sind im $\S 2$ Abs. 1, Teilzeit- und Befristungsgesetz (TzBFG) beschrieben. Personen gelten als teilzeitbeschäftigt, wenn ihre durchschnittliche Wochenarbeitszeit kürzer ist als die tarifliche und firmenübliche Arbeitszeit pro Woche (Himmen 2019, S. 32).

Herausforderungen zunehmender Komplexität und Agilität: Der zunehmende Transformationsdruck ist sowohl in Wirtschaftsunternehmen als auch in Organisationen im Gesundheits- und Bildungswesen oder in der öffentlichen Verwaltung deutlich zu spüren (v. Ameln 2018, S. 9). Unter dem Akronym VUKA (Volatilität, Unsicherheit, Komplexität und Ambiguität) werden nicht nur Trends der Arbeitswelt, sondern der Gesellschaften und gesamtwirtschaftlichen, globalen Umwelten verstanden (Volk 2019, S. 47). „Führungskräfte sind einerseits Schlüssel für die Bewältigung des Wandels, andererseits sind sie selbst diesem Wandel unterworfen“ (v. Ameln 2018, S. 9). Fluide Organisationsformen gelten als Antwort auf Herausforderungen agiler Arbeitswelten (Endres und Weibler 2019, S. 1). „Hinzu kommt der Kampf um hochqualifizierte Talente, die nach sinnstiftenden Partizipations- und Entfaltungsmöglichkeiten suchen“ (ebd.). In aktueller Literatur wird teilweise das Ende von Führung und Vorgesetzten postuliert (Laloux 2015). Andererseits wird ein immer größerer Bedarf an Führung konstatiert als Systemleitung, die das Handeln der Systemmitglieder so ausrichtet und koordiniert, dass das Überleben des Systems gesichert ist (v. Ameln 2018, S. 12). „Komplexität, Geschwindigkeit und Spezifika aktueller Geschäftsmodelle bringen die bisherigen Organisationsmuster - also die etablierten Machtkonstellationen und Entscheidungswege, auch mit all ihren Reformationsversuchen - an den Rand ihrer Leistungsfähigkeit. Und die Beschäftigten an den Rand der Belastbarkeit" (Volk 2019, S. 47).

\section{Theoretische Konzepte von Doppelspitzen}

„Doppelspitze“ oder im Englischen „Dual Leadership“ ist ein Oberbegriff für Führungsmodelle, bei denen zwei Personen zusammen Führungseinfluss ausüben. In der Literatur finden sich hierfür Bezeichnungen wie Co-Leadership, duale Führung, verteilte Führung, Führungsduos, Führungstandems und Topsharing. Der kleinste gemeinsame Nenner der unterschiedlichen Ausprägungen besteht im gemeinsamen Ausüben der Führung. Der Führungseinfluss geht von zwei Personen aus und erfolgt kombiniert. Das bedeutet, dass sich die Führungsaktivitäten der Einzelnen gegenseitig bedingen und sich Führungsrollen überlappen (Endres und Weibler 2019, S. 5). Im Folgenden werden wesentliche theoretische Formen, Konzepte bzw. Modelle von Doppelspitzen vorgestellt, die in der Literatur unterschieden werden.

\subsection{Hybride Tandems, Führungsdual}

Hybride Führungstandems bestehen aus zwei Personen mit unterschiedlicher Profession, in der Regel auch auf unterschiedlichen Positionen, aber auf der gleichen Hierarchieebene. Diese Leitungsmodelle finden sich häufig in Expertenorganisationen wie z.B, Hochschulen, in Sozialunternehmen und in Kreativ-Organisationen 
(vgl. Fröse und Bauer 2019; Endres und Weibler 2019). Hier werden die wissenschaftliche bzw. fachliche Leitung und die betriebswirtschaftliche Leitungsfunktion durch zwei Personen mit den entsprechenden Professionen auf der obersten Führungsebene vertreten, z.B. medizinische und kaufmännische Direktor/innen einer Klinik. „Permanent müssen hier unterschiedliche professionsorientierte Rationalitäten eingebracht werden“ (ebd., S. 8). Neben den funktionalen Spannungsfeldern von inhaltlicher Gestaltung und Wirtschaftlichkeit sind meistens ein unterschiedlicher Habitus, unterschiedliche Kapitalien (Ökonomie, Bildung, Netzwerke), unterschiedliche Identitäten und unterschiedliche Sichtweisen auf die Organisation (vgl. Fröse und Bauer 2019, S. 138) bei den Führungskräften vorhanden.

Fröse und Bauer (2019, S. 140) beschreiben daher aus dem Blickwinkel von Pierre Bourdieus Feld- und Habitustheorie, dass für ein hybrides Leitungsmodell eine flankierende Beratung in Form eines Coachings unabdingbar ist, wenn die Mitglieder der Doppelspitze sich in den Strukturen, verborgenen Dynamiken und Spannungen nicht aufreiben, entwerten oder vertreiben wollen. Im Fokus sollten dabei nicht die Biografien, Persönlichkeiten und Probleme des Führungsduals stehen, sondern die Felddynamik, die habituellen Prägungen, die Normalität der Konkurrenz sowie die neuralgischen Punkte der Organisation, die das Zusammenspiel bestimmen und prägen. Sie schlagen vor, ,, die üblichen Diskussionen um die narzisstischen Persönlichkeitsanteile erfolgreicher Leader hintanzustellen, obwohl es diese natürlich auch genügend gibt“, und zu versuchen, diese im Modell liegenden Spannungen feld- und habitusspezifisch zu erklären (ebd., S. 140).

Reid und Karambayya (2009) haben sich in ihrer qualitativen Forschung zu hybriden Führungsdualen in acht Kreativ-Organisationen in Kanada auf die Konfliktdynamik, ihre Auswirkungen und Bearbeitungsstrategien unter sozialpsychologischer Perspektive konzentriert. Sie halten grundsätzlich Organisationen mit Führungsdualen für kreativer, demokratischer und schneller bei Veränderungen der Umwelt. Sie beziehen sich dabei auf Pearce und Sims (2002) und Gronn (2002). Voraussetzung ist, dass Pflichten und Arbeitsteilung ausgehandelt werden und die Führungsaufgabe als gemeinsam wahrzunehmende Rolle verstanden wird. Im Ergebnis zeigten sich die üblichen Phänomene, die in der Konfliktforschung unter zwei Parteien bekannt sind. Entscheidend war jedoch, welche Auswirkungen diese auf die Organisation und ihre Mitglieder hatten. In den Aushandlungsprozessen und Konfliktszenarien wurden die Paradoxien deutlich, die in der Gesamtorganisation vorzufinden sind (Reid und Karambayya 2009, S. 1094). „Die Doppelspitze als Leitung einer Organisation steht also positioniert im Raum, der ein Ensemble von Kräfteverhältnissen ist, denen man sich nicht entziehen kann“ (Fröse und Bauer 2019, S. 137).

\subsection{Mixed Leadership}

Aktuell in der Öffentlichkeit wahrgenommene Modelle von Doppelspitzen weisen sich durch einen geschlechtsspezifischen Faktor aus. Sie sind meist explizit wie bei BÜNDNIS 90/DIE GRÜNEN, SPD und Die Linke als Frau-Mann-Duo konstruiert. Realität ist, dass die obersten Führungsebenen habitusbetonter Organisationen nach wir vor, trotz aller Selbstverpflichtungen und Frauenquoten, männlich besetzt sind. Wird die Doppelspitze durch ein Frau-Mann-Team vertreten, kommen zu den bisher 
genannten Herausforderungen doppelter Führung auch genderspezifische Aspekte. Wissenschaftliche Untersuchungen zur Mixed Leadership bezogen auf Führungstandems fehlen jedoch gänzlich. So möchte ich auf ein paar allgemeine Aspekte aus der Zusammenarbeit von gemischten Männer- und Frauenteams hinweisen.

Moderne Unternehmen sehen die Chance und verstehen es, weibliche und männliche Stärken gleichermaßen zu nutzen (Weber et al. 2016, S. 295). Dies sollte insbesondere für gemischte Führungsteams gelten, wobei natürlich sowohl Frauen als auch Männer unterschiedliche Anteile an sogenannten weiblichen und männlichen Kompetenzen haben. Bei „Mixed Teams“ kommen die Zuschreibungen von außen und von innen hinzu, z. B. durch rollentypische Presseanfragen. Darüber hinaus bietet ein Frau-Mann-Führungsteam immer auch eine besondere Übertragungsfläche für Mutter- und Vaterrollen (vgl. Schreyögg 2013). „Im Vordergrund steht dabei das Modell des ,Mixed Leadership', das sich als Chance für eine menschlichere, innovativere und gleichzeitig produktivere Arbeitswelt für Mann und Frau auf Augenhöhe begreift“ (Süßmuth 2009, in Fröse und Szebel-Habig 2009, S. 9).

\subsection{Topsharing, Duale Führung, Co-Leadership}

Eine besondere Form von Doppelspitzen stellen die Führungsmodelle in „Teilzeit“ dar. „Topsharing“ ist dabei der modernste Begriff, eine sogenannte Wortneuschöpfung aus „Top“ für ,Top-Management“ und ,Jobsharing“ für das Teilen einer Stelle (vgl. Kuark 2003). Duale Führung (Endres und Weibler 2019), Co-Leitung (Ellwart et al. 2016) und Führung in Teilzeit (Karlshaus 2016) werden häufig als synonyme Begriffe verwendet. In der Neuseeländischen Studie von Vine et al. (2008) werden darüber hinaus auch „Quasi-Doppelspitzen“ (Schreyögg 2005a), z. B. CEO - CFO, sowie andere Modelle von Führungskraft und ,,außergewöhnlich starkem Stellvertreter" (Vine et al. 2008, S. 2) unter Co-Leadership gefasst. Alle Modelle bezeichnen damit ein Führungsmodell, bei dem sich zwei Führungskräfte eine Managementposition teilen (Ellwart et al. 2016, S. 253). Hier liegt eine vollständige oder sehr breite Rollenüberlappung vor (Endres und Weibler 2019, S. 6). Dabei kann es sich um klassische Teilzeit oder einfach auch um zwei Personen handeln, die sich aus Gründen von Umfang, Komplexität oder aus strategischen Gründen wie einer Fusion „eine" Stelle/Position teilen, z.B. die Position der Vorstandsvorsitzenden.

Himmen hat 2019 erstmalig quantitativ belastbares Datenmaterial zur Praxis von Topsharing in deutschen Unternehmen erhoben. Es zeigte sich, dass Topsharing bei Frauen und Männern von großem Interesse ist. Die Unterschiedsdifferenz $(r=29)$ des höheren Interesses von Frauen ist gering (Himmen 2019, S. 13). Ein kinderabhängiger Effekt kann statistisch nicht als signifikant bestätigt werden (ebd., S. 13). „Nicht mehr der heroische Leader ist hier gefragt, sondern vielmehr braucht es zwei Persönlichkeiten, die über hohe soziale Kompetenzen verfügen“ (ebd., S. 7). Neben den sozialkompetenzorientierten Vorteilen, die zukünftig auch für Mitarbeitendengewinnung und Bindung zentral sein werden, beschreibt Himmen den Diversitäten fördernden Charakter als Mehrwert. Als besondere Herausforderung sieht sie, dass Rollenvorbilder entstehen können (ebd., S. 7). In ihrer Untersuchung hat sie neben den Interessen aktiver und zukünftiger Topsharing-Personen auch Persönlichkeitsfaktoren nach dem Big-Five-Modell (Barrick und Mount 1991) erhoben. Auch wenn 
hier kein signifikanter Einfluss nachgewiesen wurde, führt eine gesteigerte Problemlösungskapazität, die aus einer gegenseitigen Kompetenzergänzung resultiert, beim Topsharing zu einer verbesserten Führungsleistung (Himmen 2019, S. 126).

Von besonderer Bedeutung ist in diesem Führungsmodell mit hohen gemeinsamen Führungsanteilen die Kommunikation. In einer neuseeländischen empirischen Studie von Vine et al. (2008) wurde in Fallstudien das Kommunikationsverhalten von Co-Leitungen in drei Unternehmenskontexten, die als besonders führungsstark galten, untersucht. Als Schlüsselkomponente wurde die Art, wie Beziehungen durch Gespräche ausgehandelt werden, erforscht, wie Co-Leitungen gemeinsame Gespräche mit ihren Mitarbeitenden und Gremien führen und wie die Position des Führungstandems gestärkt wird. Ergebnis war, dass erfolgreiche Co-Führer/innen sehr kontextbezogen agieren und sich auf ein dynamisches Zusammenspiel einlassen (ebd., S. 354). „Praktisch geht es beispielsweise dann darum, wie sich zwei Personen, die gemeinsam Führungsverantwortung für ein Team haben, etwa in einem Meeting, geschickt Bälle zuspielen, um Einfluss auf die Gruppenteilnehmer auszuüben. Dies kann so diskret erfolgen, dass im Einzelnen gar nicht festgelegt werden kann, von wem der letztlich erwirkte Einfluss auf die Gruppe ausging“ (Endres und Weibler 2019, S. 7). Vine et al. (2008, S. 355) empfehlen daher, der alltäglichen Kommunikation z. B. bei den täglichen/wöchentlichen Besprechungen erhöhte Aufmerksamkeit zukommen zu lassen und den diskursiven, wechselseitigen Aspekt auch in Führungskräftetrainings mehr Beachtung zu schenken.

\section{Der Blick hinter die Fassade: Qualitative Untersuchung zu Gelingensfaktoren und Herausforderungen von Doppelspitzen}

Die Recherche vorhandener Literatur- und Forschungsergebnisse zeigte, dass dort häufig die erwünschte Schauseite des Konstrukts Doppelspitze abgebildet wird. Mein Interesse war, in einem zweiten Schritt den Blick hinter die Fassade zu werfen und zu erforschen, wie eine stabile Struktur in Doppelspitzen entsteht und wie Herausforderungen und Scheiternsfaktoren konkret aussehen. Ich führte im Februar 2020 sechs leitfadengestützte qualitative Experteninterviews, nicht mit den Doppelspitzen direkt, sondern mit sieben Coaches, die mehrjährige Erfahrung in der Beratung von Führungstandems haben. Zwei Coaches wurden gemeinsam befragt, da diese häufig im Tandem Doppelspitzen beraten. So entstand auf einer Meta-Ebene ein Einblick in die Arbeit von ca. 120 Doppelspitzen mit einer großen Bandbreite der Branchen von DAX-Unternehmen über Start-Ups, Non-Profit-Organisationen bis hin zu Familien- und Handwerksunternehmen. Die Interviews wurden durch eine qualitative Inhaltsanalyse nach Mayring (2010) ausgewertet.

\subsection{Persönliche und fachliche Passung der Tandems}

Die Ergebnisse der Interviews zeigen, dass der persönlichen und fachlichen Passung die höchste Bedeutung beigemessen wird. Dabei werden sowohl individuelle Faktoren, z. B. intrinsische Motivation, Offenheit, Reflexionsfähigkeit, Selbsterkenntnis, 
Stress- und Affektkontrolle, als auch interaktive Kompetenz, z. B. Kommunikationsund Problemlösungs- und Diversitätskompetenz, als wesentlich erachtet.

Die Befunde sind ebenso in der Forschung zu Teamarbeit zu verorten. Als Faktoren, die die persönliche Passung beeinflussen, wurden in den Interviews zum einen individuelle Merkmale wie Skills, Wissen, Fähigkeiten, Erfahrungen, Persönlichkeitsmerkmale genannt, die auch in der Forschung zu Teamarbeit als Gelingensfaktoren beschrieben sind (z. B. Guzzo und Dickson 1996). Zum anderen scheinen die Team-KSAs (knowledge, skills, abilities) wie Konfliktlösungsfähigkeit, kollaborativer Problemlösestil, Kommunikationskompetenz, Selbstmanagement, wie sie wissenschaftlich bei Stevens und Campion $(1994,1999)$ beschrieben sind, von hoher Bedeutung. Es ist davon auszugehen, dass empirische Erkenntnisse aus dem Bereich der Teamforschung auch auf die Zweierkonstellation übertragbar sind, die besagen, dass neben den hohen Fähigkeiten der Einzelnen die Team-KSAs einen zusätzlichen Vorhersagewert für die Gelingensfaktoren für Doppelspitzen darstellen.

Die Ergebnisse der Interviews können darüber hinaus dahin interpretiert werden, dass durch eine Verdichtung der Zusammenarbeit in der Zweierkonstellation die individuellen Fähigkeiten und interaktiven Kompetenzen eine noch wesentlich größere Rolle spielen als in Teams ab drei Personen. Dies lässt sich sehr eindrücklich aus folgendem Zitat einer Coach schließen: „Zentrale Herausforderungen an Doppelspitzen sind (...) die enge Zusammenarbeit, wo man mit seinen ganzen persönlichen Störungen oder positiv formuliert mit seinen persönlichen Entwicklungsthemen aufeinanderprallt." Umso erstaunlicher ist es, dass bei der Personalauswahl von Doppelspitzen kaum eine Beratung stattfand; es wurde meistens kein spezielles Personalauswahl-Verfahren eingesetzt. Auch dieser Aspekt wurde bereits in der kanadischen Studie von Reid und Karambayya (2009) bemängelt, dass Personalauswahl und Passung einer Doppelspitze von den Aufsichtsgremien kaum bedacht werden. Instrumente der Personaldiagnostik, wie z. B. die Operationalisierte Psychodynamische Diagnostik im Arbeitskontext (Kotte et al. 2019) und das Bochumer Inventar zur berufsbezogenen Persönlichkeitsbeschreibung (Hossiep und Paschen 2003) könnten für die Auswahl von Doppelspitzen weiterentwickelt werden.

\subsection{Diversität und Komplementarität nutzen}

Unterschiede und damit verbundene Komplementarität wurden von den meisten befragten Expert/innen als wesentliche Vorteile für die Potentialentfaltung einer Doppelspitze beschrieben. Die Komplementarität erstreckt sich sowohl auf Charaktereigenschaften als auch auf Professionen, Kompetenzen und Geschlechter. Es gibt jedoch auch Unterschiede, die sich nachteilig auswirken und eine Zusammenarbeit auf Augenhöhe stark erschweren, wie z.B. zu große Altersunterschiede. Auch asynchrone Entwicklungen des Duos, z. B. im Gesundheitszustand und bei Leistungsunterschieden, führen gehäuft zu schwer lösbaren Konflikten. Die befragten Coaches halten es daher für wesentlich, das Konstrukt „Doppelspitze“ mit seinen Komplementaritäten immer wieder für die Doppelspitzen-Mitglieder selbst, aber auch in die Mitarbeitendenschaft hinein zu kommunizieren. Dies beschreiben ebenso Hartmann et al. (2013), die die Kommunikation der Vorteile von Heterogenität in die Belegschaft als notwendig betonen. Einen wesentlichen Einfluss auf den Nutzen 
von Diversität in der Doppelspitzenarbeit hat dabei auch deren Wertschätzung durch das unternehmerische Umfeld und die Organisationskultur (vgl. Hockling 2019).

Fröse und Bauer (2019) sowie Endres und Weibler (2019) sehen in den Unterschieden von Habitus, Kapitalien, Professionen, Identitäten und Sichtweisen eher Spannungsfelder, die bei (unbewusst verborgenen) Dynamiken zu Entwertungen und Reibungsverlusten führen. Endres und Weibler schreiben dann aber weiter, dass Diversitäten des Duos als strategisch gewollt implementiert werden müssen, dann seien sie hilfreich. Hartmann et al. (2013) gehen noch einen Schritt weiter, indem sie postulieren, dass die Vorteile der Heterogenität in der Belegschaft kommuniziert sein müssen. Die Ergebnisse aus den bisherigen kleinen außereuropäischen Studien werden auch an diesem Punkt bestätigt. Die inkonsistenten Forschungsergebnisse aus zahlreichen Einzelstudien und Überblicksarbeiten zwischen Teamdiversität und Teamerfolg zeigen sowohl positive wie negative, aber auch keine Zusammenhänge (Bell et al. 2011; Joshi und Roh 2009; Kearney und Voelpel 2012; Thatcher und Patel 2012; van Knippenberg und Schippers 2007; van Knippenberg et al. 2004).

In dieser qualitativen Untersuchung wurden jedoch Zusammenhänge und gegenseitige Einflussfaktoren deutlich, die insbesondere von einer entsprechenden Unternehmenskultur geprägt sind: Agile, wissensbasierte Unternehmen fördern und wertschätzen die Unterschiede für den unternehmerischen Erfolg. Dies ist in der Regel verbunden mit einer jüngeren Generation an Fach- und Führungskräften, die ihre Individualität und besonderen Kompetenzen bereits differenziert in den Blick nehmen und im beruflichen Kontext auch einsetzen wollen (vgl. Abdul-Hussain 2012).

Wenn Hartmann et al. (2013) schreiben, dass bei einem ausgeglichenen Geschlechterverhältnis in der Branche weniger Stereotypisierungen erfolgen, ist nach den vorliegenden Interviews davon auszugehen, dass dieser Umstand auch auf das Verhältnis von unterschiedlichen Berufsgruppen anzuwenden ist. Vor allem in den Bereichen, in denen ein ausgeglichenes Zusammenwirken von unterschiedlichen Berufsgruppen stattfindet (z. B. zwischen Betriebswirt/innen und Techniker/innen), können die Potenziale beruflicher Diversität besonders gut genutzt werden. Ebenso ließen die Interviews darauf schließen, dass in traditionellen und hierarchischen Organisationen an diesen Stellen gehäuft eine Störanfälligkeit auftritt.

\subsection{Mehrwert für Beteiligte und Unternehmen - ein zentraler Schlüsselaspekt}

In allen Interviews wurde deutlich, dass ein entscheidender Gelingensfaktor darin liegt, dass sowohl für die Menschen, die sich auf das Führungsmodell „Doppelspitze“ einlassen, als auch für das Unternehmen, seien es die Aufsichtsgremien und/ oder Mitarbeitende, ein deutlich spürbarer Mehrwert durch dieses Führungskonstrukt entstehen muss. Folgende Aspekte wurden genannt:

- Mehrwert für Unternehmen: Höhere Leistungsfähigkeit für die Organisation, bessere Entscheidungen bei komplexen Sachlagen, Steigerung der Arbeitgeberattraktivität, Gleichstellung von Frauen und Männern auf Führungsebene, Ermöglichung von mehr Wachstum und Einflussnahme, Verhinderung von Machtmissbrauch und Möglichkeit der Nachfolgeregelung. 
- Mehrwert auf der individuellen Ebene: Work-Life-Balance, Ermöglichung von Familienarbeit und Führungsposition, Freiräume für berufliche Leidenschaften und „Lieblingsprojekte“, gegenseitige Unterstützung und Entlastung in komplexen und schwierigen Situationen.

Diese Befunde waren zunächst überraschend. Der Indikator „Mehrwert“ ist in der aktuellen Forschung und Literatur nicht explizit dargestellt, bietet jedoch in den Interviews einen deutlichen Dreh- und Angelpunkt. Hier steckte bei den Gelingensfaktoren am meisten Emotionalität und eigene Beteiligung der befragten Expert/ innen. Es scheint durchaus einleuchtend, dass Herausforderungen, z. B. das Teilen von Macht und ein daraus resultierender erhöhter Abstimmungs- und Kommunikationsbedarf, durch positive Effekte ausgeglichen oder besser noch deutlich übertroffen werden sollten. Dabei ist aus meiner Sicht zu beachten, dass durch die enge Zusammenarbeit und das gemeinsame Führen eines Unternehmens(-teils) eine dritte Größe entsteht. Wo das dyadische Denken um eine Balance zwischen Nähe und Distanz, um ein Oszillieren zwischen Verschmelzung und Autonomie ringt, entsteht durch das Dritte ein triadischer Raum. Das zweiwertige Denken wird erheblich differenziert und führt eben zu diesem (Busse und Tietel 2018, S. 16). Das Dritte ist hier z.B. die primäre Aufgabe der gemeinsamen Führung. Dadurch kann für komplexe Aufgabenstellungen ein Mehrwert durch Führungstandems (bei größeren Organisationen durch Führungsteams) entstehen.

Die Forschungssynopse zu geteilter Leitung von Endres und Weibler (2019) stellt in ihren Befunden eine Vielzahl von positiven Wirkungen für Individuen, Organisation und Gesellschaft dar. Die Auseinandersetzung mit den Vorteilen und Zielen von Doppelspitzen sowie das Herausarbeiten positiver Bilder scheinen an der Schnittstelle von Führungsforschung und Coachingkonzepten zielführend.

\subsection{Gemeinsames Führungsbild und klare Aufgabenteilung}

Als weiteren Gelingensfaktor werden die Entwicklung eines gemeinsamen Führungsbildes, die Abstimmung von Vision, Mission und Strategien, die Rollen- und Aufgabenklärungen einschließlich der Verhandlung persönlicher Interessen gesehen. Dabei wurden ein abgestimmtes Führungsverständnis und ein gemeinsames Auftreten als Basis bzw. bei deren Fehlen als Scheiternsfaktor beschrieben. Diese Faktoren bilden somit ein Fundament für Führungshandeln zu zweit. Häufig sind formale Regelungen wie Geschäftsverteilungspläne vorhanden oder werden in einer Startphase entwickelt oder überprüft, ergeben jedoch allein noch kein gemeinsames Führungshandeln. Häufig wird Führung noch zu sehr als Einzelleistung eines Managers gesehen und weniger als orchestrales Werk eines Führungsteams. Das Potenzial der Doppelspitzenarbeit entsteht nicht durch die Multiplizierung von Leitungsstellen, sondern durch ein anderes Führungsverständnis (vgl. Endres und Weibler 2019; Hinz 2014). Um dieses zu erreichen, ist ein differenzierter und stets reflektierender Aushandlungsprozess des Führungstandems notwendig. Bei einem positiven Verlauf werden dadurch eine erhöhte Motivation und damit eine erhöhte Leistung des Führungsduals wahrgenommen. Dies wird in der Regel durch eine hohe Identifikation mit dem gemeinsamen Konstrukt „Doppelspitze“ in Verbindung mit der 
Primäraufgabe der Organisation erreicht. Dieser Umstand wird in der Organisationspsychologie unter dem Begriff „Social Laboring“ beschrieben (Worchel et al. 1998).

\subsection{Einfluss von Dritten und Auswirkungen der Felddynamik}

$\mathrm{Zu}$ einer der größten Herausforderungen von Doppelspitzen zählten der Einfluss von Dritten, z. B. von Mitarbeitenden oder Aufsichtsräten, sowie die Auswirkungen der Felddynamik. In den Interviews wurden im wesentlichen direkte Störungen durch Mitarbeitende, Vorgesetzte, Kunden oder Familienmitglieder beschrieben, so z. B. Kunden, die nur den älteren Steuerberater aufsuchten, oder Mitarbeitende, ,die wie die Kinder ihre Eltern“ das Führungsduo gegeneinander ausspielten. Interessanterweise waren selbst diese offensichtlichen Dynamiken den Doppelspitzenpartner/ innen nicht bewusst. Sie suchten auch in diesen Fällen Coaching auf, um die Beziehungs- und Arbeitsebene zu klären, sahen also nur einen dyadischen und keinen triadischen Konflikt.

In der Literatur wird dies ebenso als neuralgischer Punkt beschrieben. So sehen Fröse und Bauer (2019), dass die Felddynamik der Organisation und deren Umfeld die Zusammenarbeit der Führungsduos erschweren, indem Konkurrenz und (unbewusste) Spannungsfelder auf das Führungsduo übertragen werden. Reid und Karambayya (2009) konnten in ihrer Studie in der kanadischen Kreativbranche feststellen, dass in den Konfliktszenarien der Doppelspitze häufig Paradoxien deutlich werden, die in der Gesamtorganisation vorzufinden sind.

\subsection{Sorgfältige Implementierung}

Der Anteil der befragten Coaches ist äußerst gering, die Organisationen oder Doppelspitzen im Vorfeld und zu Beginn ihrer Tätigkeiten beraten. Dennoch sehen die Coaches einen sehr hohen Faktor für das Gelingen in diesem Bereich: Ein deutliches Abwägen von Vor- und Nachteilen eines Doppelspitzenkonstrukts, ein Herausarbeiten, welche Ziele und Visionen mit der Doppelspitze verbunden sind, eine entsprechende Kommunikation in die Organisation und nach außen, eine Begleitung zum Start sowie die bereits beschriebenen Faktoren - von der Personalauswahl bis zum gemeinsamen Führungsbild - sind vor der Implementierung zu bedenken.

Endres und Weibler (2019, S. 27) betonen als Fazit ihrer Forschungssynopse: „Eine nachhaltige Entfaltung des positiven Potenzials von Plural Leadership hängt von der Art und Weise seiner Einführung ab.“ Auch andere Autoren verweisen auf die Notwendigkeit einer guten Einführung und Begleitung in der Startphase (Fröse und Bauer 2019; Himmen 2019; Ellwart et al. 2016; Reid und Karambayya 2009; Wimmer 2004). Allerdings ist dieser Aspekt weder in der Forschung näher betrachtet noch in der Führungs- und Coachingpraxis konzeptionell beschrieben worden.

\subsection{Raum für Reflexion und Beratung}

Zeitliche Ressourcen und persönliche Bereitschaft zu einer intensiven Zusammenarbeit, Reflexion und Auseinandersetzung bilden die Basis für Führungstandems, 
so die zentrale Aussage der Expert/innen. Der hohe Abstimmungsbedarf von Führungstandems wird immer wieder als negativer Faktor beschrieben, zuletzt auch beim Scheitern der Doppelspitze bei SAP: Die Krise brauche schnelle Entscheidungen durch einen starken Mann. Endres und Weibler (2019) verstehen jedoch das Ringen um Verständigung nicht als negativen Kostenfaktor, sondern als förderlichen Entstehungsfaktor, der einen Lern-Dialog kultiviert. Ähnlich sehen dies Reid und Karambayya (2009) und v. Ameln (2015). Offene Aushandlungsprozesse wirken gleichzeitig klärend und kulturbildend für die Organisation, indem Polaritäten, Zielkonflikte und Ambiguitäten nicht auf Nebenschauplätze verschoben werden. Bei der Gestaltung von Form und Maß der Zusammenarbeit sind aber ebenso die paradoxen Effekte zur Teamreflexivität nach Schippers (2012) zu berücksichtigen, die besagen, je weniger Zeit und/oder Ressourcen Teams haben, um zu reflektieren, desto deutlicher sind die Effekte der Reflexivität (Schippers et al. 2015).

\subsection{Angst vor einem Scheitern und deren Konsequenzen}

Als besonders sensibler Aspekt zeigte sich die Angst vor dem Scheitern der Doppelspitze. Sie wirkt sich sowohl auf die Doppelspitzen selbst als auch auf die Organisation aus. Zum einen hat ein Scheitern der Zusammenarbeit in vielen Bereichen, z.B. bei Freiberuflern oder Familienunternehmen, auch weitreichende persönliche und wirtschaftliche Konsequenzen. Zum anderen wurde gerade in den Interviews deutlich, dass mit dem Scheitern eines Führungsduos Konflikte in der Organisation offensichtlich werden und damit oft die ganze Organisation in Frage gestellt ist.

Der Effekt, dass das Scheitern der Führungsduos die Organisation oft in eine existenzielle Krise stürzt, wurde ebenfalls in der Studie von Reid und Karambayya (2009) und bei Himmen (2019) festgestellt. Ellwart et al. (2016) empfehlen daher bei der Einführung einer Doppelspitze, insbesondere im Topsharing, eine zeitliche Befristung. Reid und Karambayya sehen es dagegen als kritisch, insbesondere wenn unterschiedliche Vertragslaufzeiten, z.B. bei Vorstandsverträgen, die Regel sind. Meines Erachtens ist mit einer zeitlichen Befristung sehr vorsichtig umzugehen, wenn wir uns vor Augen führen, welche Auswirkungen „Dritte“, z. B. Aufsichtsgremien und Mitarbeitende, auf das Führungsduo haben. Zeitlich befristete Konstrukte können hier eine schwierige Dynamik schneller befeuern, als dass sich daraus positive Effekte entwickeln.

\section{Konzeptionelle Eckpunkte für Doppelspitzen-Coaching}

Im Folgenden werden Eckpunkte für ein Coaching formuliert, welche den aktuellen Forschungsstand und die Ergebnisse der qualitativen Experteninterviews aufnehmen. Auf die Spezialbereiche „Coaching zur Trennung von Doppelspitzen“ sowie „Coaching zur Nachbesetzung eine/r Doppelspitzenpartner/in“ wird dabei nicht eingegangen. Der Bereich „Konfliktcoaching und Mediation“ von Doppelspitzen ist bereits bei Schreyögg (2005b) und Fildhaut (2013) beschrieben. 


\subsection{Phase der Vorüberlegungen}

Im Vorfeld der Einführung des Führungsmodells Doppelspitze sind Beratungen auf folgende Aspekte zu richten:

- Ziele und beabsichtigter Mehrwert des Führungsmodells „Doppelspitze“: Ziele und Vorstellungen, die mit einer Doppelspitzenstruktur verbunden werden, haben einen wesentlichen Anteil auf die spätere Funktion des Führungsmodells. Insbesondere über den erhofften Mehrwert für die Organisation und die beteiligten Führungskräfte sollte offen gesprochen und reflektiert werden. Dabei ist zu bedenken, dass insbesondere bei komplexen, neuartigen Problemstellungen sowie in wissensbasierten Kontexten dieses Führungsmodell wesentliche Vorteile hat (Endres und Weibler 2019, S. 42). Coachingthemen: Anlass, über veränderte Führungsmodelle nachzudenken; Ziele der Doppelspitze; Fokussierung auf den Mehrwert, interne und externe Kommunikation des Mehrwerts.

- Organisationskultur auf Offenheit bzw. Veränderungsbereitschaft überprüfen: „Der ,kulturelle Ansatz“ gilt heute als einer der wichtigsten Zugänge zur Erforschung von Organisationen“ (Giernalczyk und Möller 2018, S. 81). Da diese Muster für die Mitglieder einer Organisation im Wesentlichen implizit bleiben, sind vor der Veränderung einer Führungsstruktur die Bereitschaft und Offenheit der Organisation und ihrer Kultur für neue Führungsbilder - weg von der alleinigen Führungsspitze - zu prüfen und zu entwickeln. Hilfreiche Fragen zur Organisationskultur: Welche Normen und Standards liegen in der Organisation vor? Versteht man sich eher als Familie oder in professioneller Distanz? Welche Bilder von Führung sind damit verbunden? Wird Führung als Organisationsfunktion gesehen oder wird sie individualisiert? Ist eine Abkehr von der One-ManShow zu orchestraler Führung denkbar? Wie viel Entwicklungsraum wird den Mitarbeitenden zugebilligt? Wie werden Entscheidungen getroffen, partizipativ oder autoritär? Herrscht Wettbewerb oder Kooperation in der Organisation? Über welche Themen darf nicht gesprochen werden?

\subsection{Coaching in der Implementierungsphase}

- Mehrwert durch gemeinsame Handlungsfelder und individuelle Aufgaben des Führungsduals entwickeln: Diese können z. B. in einem Geschäftsverteilungsplan beschrieben und mit entsprechenden Kompetenzanforderungen für die Personalauswahl formuliert werden. Coachingthemen: Entwicklung positiver Bilder von Doppelspitzen; persönliche Stärken und fachliche Schwerpunkte des Duos zu einem gemeinsamen Werk entwickeln; Entscheidungs- und Entwicklungsraum jedes Einzelnen bedenken.

- Auswahl der Führungskräfte für die Doppelspitze: Dabei sind Aspekte wie Komplementarität von Ausbildungshintergründen, Kompetenzen der Zusammenarbeit sowie erforderliche psychische Basisfähigkeiten und zentrale motivationale Lebensthemen (Kotte et al. 2019) gleichermaßen zu beachten. Unterstützende Methoden bei der Personaldiagnostik können insbesondere die Operationalisierte 
Psychodynamische Diagnostik im Arbeitskontext (ebd.) und das Bochumer Inventar zur berufsbezogenen Persönlichkeitsbeschreibung (Hossiep und Paschen 2003) sein.

- Entwicklung eines gemeinsamen Führungsbildes: Bei gelingenden Doppelspitzen geht es nicht um die Teilung oder Multiplizierung von Leitungsstellen, sondern um ein grundlegend andersartiges Führungsverständnis (Endres und Weibler 2019). Dieses umfasst nicht nur das Führungstandem, sondern auch die Gesamtorganisation und impliziert eine Vertrauenskultur (Möller 2012). Folgende Themen sind zu beleuchten: Arbeit an Führungsbildern, z. B. auch mit Metaphern und anderen kreativen Medien; Reflexion der Praktiken und Vorgehensweisen bei der Zielerreichung; Sensibilisierung für Interdependenzen; Orientierung an der Primäraufgabe der Organisation und Abwägen des primären Risikos.

- Kommunikation des Führungsmodells: Ziele des Führungsmodells und gewollte Unterschiede, die Diversität des Duos werden nach innen und nach außen kommuniziert.

- Besprechungs- und Reflexionszeiten: Es werden regelmäßige Besprechungszeiten implementiert, in denen neben strategischen und fachlichen Absprachen die Reflexion der Zusammenarbeit ein steter Punkt auf der Tagesordnung ist.

- Monatliches Coaching des Führungstandems: Im ersten Jahr der Zusammenarbeit sollte ein monatliches gemeinsames Coaching des Führungstandems wahrgenommen werden. Dabei darf aber auch im Doppelspitzen-Coaching nicht vernachlässigt werden, dass die Steigerung der persönlichen Kompetenzen - wie sie auch sonst im Coaching bedacht wird - einen zentralen Einfluss auf das Gelingen der Führungstandems hat. Daher können auch Einzelcoachings zur Bearbeitung persönlicher Themen der Führungskräfte sinnvoll sein.

\subsection{Regelmäßig begleitendes Doppelspitzen-Coaching}

- Auch wenn eine Doppelspitze gut implementiert und eingearbeitet ist, gilt es immer wieder, einen Raum für die regelmäßige Reflexion der Zusammenarbeit, der Gelingensfaktoren und der Herausforderungen zu schaffen. Im Coaching sind hier insbesondere folgende Aspekte relevant:

- Zwischen Aufgabenklarheit und Rollenflexibität-neue Muster der Zusammenarbeit entwickeln, Sicherheit und Dynamik ausbalancieren: Aufgabenklarheit und komplementäres Agieren sind grundsätzlich zu fördern und gleichzeitig daraufhin zu prüfen, inwieweit entstandene Muster zu einer Erstarrung und Rollenfestlegung führen. Es gilt, eine flexible und dynamische Zusammenarbeit zu entwickeln, um die in der Doppelspitze angelegte Agilität zu fördern und Wachstumsprozesse sowohl für das Individuum als auch für die Organisation zu ermöglichen. Asynchrone Entwicklungen und Rollenstarrheit werden dadurch vermieden. Regelmäßige Reflexionsschleifen und direkter Dialog sind z. B. durch die ,3-B-Regelung“ Beobachtung, Bedeutung, Bewertung zu fördern (Hinz 2014, S. 102). Psychodynamisches Coaching kalkuliert Konflikthaftigkeit, Irrationalität, Mikropolitik, Neid, Angst und Liebe mit ein (Giernalczyk und Möller 2018, S. 99). Die Bedeutung 
von Macht, individuellen Interessen und Zielen ist ebenso zu bedenken (Rastetter und Jüngling 2018, S. 11) wie die Reflexion der Aufgabenzuschnitte, die Bearbeitung von Rollenzuschreibung und Rollenflexibilität, Balance und Neujustierung des Gleichgewichts des Führungstandems. Hier sind auch die Angst vor dem Scheitern durch Reflexion der eigenen Angsttoleranz, der Umgang mit schwerwiegenden Emotionen, ein Bewusstsein für Dringlichkeit von Veränderung, systematische und regelmäßige Übung der Rollendistanz einzubeziehen.

- Führungstandems als Übertragungsfiguren - Gegenübertragungen untersuchen: Führungskräfte werden generell häufig wie Elternteile wahrgenommen, und ihre Macht wird aufgrund der Übertragung überschätzt (Giernalczyk und Möller 2018, S. 34). Führungstandems, insbesondere gemischtgeschlechtliche, ziehen noch häufiger Übertragungen und Projektionen von ihren Mitarbeitenden auf sich. Das regelmäßige Innehalten und Reflektieren der Gegenübertragung (Was habe ich gedacht und gefühlt? Welche Bilder und Handlungsimpulse sind aufgetaucht?) hilft, mit den herangetragenen Emotionen besser umzugehen (ebd., S. 35). Innerhalb des Führungstandems kann es auch zu „Geschwisterübertragungen“ kommen, die ebenso zu reflektieren sind (vgl. Schreyögg 2009, 2013)

- In triadischen Beziehungen orientieren - Triangulierung verstehen und nutzen: Der Einfluss von „Dritten“ ist eine der größten Herausforderungen für Doppelspitzen. Wie beschrieben, bildet die Entwicklung von der Führungsdyade zur Triade, z.B. durch die Orientierung an der primären Aufgabe sowie der weiteren Wahrnehmung vielfältigster triadischer Wirklichkeiten, ein Kernstück triadischen Denkens. Im Coaching von Führungstandems kann diese ,,ihren Mehrwert entfalten, wenn die komplexitätsreduzierende wie -erweiternde triadische Brille auf lebens-, arbeitsweltliche wie beraterische Interaktionen gerichtet und genutzt wird" (Busse und Tietel 2018, S. 101).

- Organisationskulturen analysieren, Eigendynamiken reflektieren, Gestaltungsräume eröffnen: Auch wenn bereits vor der Implementierung der Doppelspitze die Organisationskultur in den Blick genommen wurde, werden sich im weiteren Verlauf der Arbeit neben der Oberflächenstruktur auch fortwährend die verborgenen Grundannahmen, Normen und Werte auf das Führungstandem auswirken (Giernalczyk und Möller 2018, S. 86). Die Felddynamik beeinflusst die Dynamik der Doppelspitze in besonderer Weise. Im Coaching können Spiegelphänomene und Übertragung/Gegenübertragung für die Arbeit nutzbar gemacht werden. Eine Reflexion, die sowohl die dem Handeln der Organisationsmitglieder zugrundeliegenden Muster, Normen und Einstellungen als auch die damit verbundene Eigendynamik der Doppelspitzenmitglieder betrachtet, kann Spielräume der Veränderung definieren und sich gleichzeitig klärend und kulturbildend für Organisation und Führungstandem auswirken (vgl. Giernalczyk und Möller 2018; v. Ameln 2018; Reid und Karambayya 2009).

- Selbstwirksamkeit der Doppelspitze erhöhen: Selbstwirksamkeit gilt als Schlüsselwirkstoff sowohl für gelingendes Führungshandeln wie für die Wirksamkeit von Coaching (vgl. de Haan et al. 2016). Im Coaching von Doppelspitzen kann sowohl an der individuellen Selbstwirksamkeit als auch an der gemeinsamen Selbstwirksamkeit des Führungstandems gearbeitet werden. Die Entwicklung gemeinsamer Stories wirkt stärkend auf das gemeinsame Führungshandeln. 
- Strategien und Komplexität beidhändig bearbeiten: „Das Unbewusste in der Leitungsebene und deren biografische Erfahrungen spielen in einem Strategieprozess eine entscheidende Rolle. Das Zusammenspiel zwischen den Entscheidern prägt das Denken im Strategieprozess ebenso wie die direkte und indirekte Einflussnahme wichtiger Stakeholder“ (Nagel 2012, S. 229). Die Integration der unbewussten Aspekte in den unternehmerisch wichtigen Prozess der Strategieentwicklung führt zu Erkenntnissen, die in besonderer Weise bei Doppelspitzen den Entscheidungsund Handlungsspielraum erweitern können (Nagel 2013).

- Selbstreflexion als Voraussetzung für das Gelingen, stetes Coaching im Einzelund im Doppelsetting: Neben den individuellen Herausforderungen, denen Menschen in einer Arbeitswelt, die durch Volatilität, Unsicherheit, Komplexität und Ambiguität gekennzeichnet ist, ausgesetzt sind, kommen die erhöhte Dynamik der Führungsdyade und die damit verbundenen Spiegelungsphänomene aus Organisation und Umfeld hinzu. Die regelmäßige Reflexion des Erlebens und Handelns ist sowohl auf der individuellen Ebene wie auf der Ebene der Doppelspitze notwendig, um ihr professionelles Selbstverständnis zu erweitern, ihre Wirkmächtigkeit zu erhöhen sowie eigene mentale Zustände und die der Kolleg/in klarer beurteilen zu können. Einüben reflektierender und generativer Dialoge, Herstellen von Querverbindungen, gegenseitige Neugierde sind die Basis gelingender professioneller Beziehungen.

\section{Fazit}

Im vergangenen Jahr wurde deutlich, welch großen Einfluss gesellschaftliche Entwicklungen auf das Ansehen und die Aktualität verschiedener Führungsmodelle haben. Während zu Beginn der Arbeit im November 2019 Führungsmodelle, die die Selbststeuerung von Mitarbeitenden in Unternehmen unterstützen, boomten und damit auch Doppelspitzen als innovatives Führungsmodell gehypt wurden, hat sich durch die Corona-Krise - nicht nur bei SAP - die Bewertung von Hierarchie, Gleichberechtigung, Chancengerechtigkeit sowie Globalisierung ganz offensichtlich geändert. Häufig zitiert wird derzeit die Auffassung, dass die Krise wie ein Brennglas auf Themenfelder wirkt und vorher die Entwicklungen ohne gesellschaftliche Tiefenwirkung nur oberflächlich angegangen wurden. Das betrifft neben der Bezahlung von systemrelevanten (Frauen-)Berufen, der geschlechtergerechten Aufteilung von Familien- und Erwerbsarbeit z. B. auch die Frage, ob neue Führungsmodelle, die auf Diversität, Partizipation, Selbststeuerung und flache Hierarchien sowie Teilung von Macht setzen, wirklich gewollt sind.

Mit dieser Arbeit wurde gezeigt, dass das Führungsmodell „Doppelspitze“ positive und negative Effekte hat, zwischen denen abgewogen werden muss. Es ist weit mehr als eine Notlösung, aber auch kein Heilsbringer. Es ist nicht für jedes Unternehmen passend, aber es kann für wissensbasierte und agile Unternehmen deutliche Vorteile bringen. Das Führungsmodell birgt Herausforderungen, die eine offene Auseinandersetzung, hohe kommunikative Kompetenzen und persönliche Reife der Führungskräfte erfordern. Die Gelingensfaktoren weisen darauf hin, dass Führung durch Doppelspitzen beziehungsbezogener wird und sich Ambiguitäten 
sowie Komplexität besser bewältigen lassen. Gesteigerte Problemlösungsstrategien und gegenseitige Kompetenzergänzungen weisen für Führungskräfte, Mitarbeitende und die Gesamtorganisation positive Effekte auf und können somit ein innovatives Führungsmodell darstellen. Durch Coaching können die positiven Effekte von Doppelspitzen verstärkt und die Herausforderungen reflektiert und bearbeitet werden.

Funding Open Access funding enabled and organized by Projekt DEAL.

Open Access Dieser Artikel wird unter der Creative Commons Namensnennung 4.0 International Lizenz veröffentlicht, welche die Nutzung, Vervielfältigung, Bearbeitung, Verbreitung und Wiedergabe in jeglichem Medium und Format erlaubt, sofern Sie den/die ursprünglichen Autor(en) und die Quelle ordnungsgemäß nennen, einen Link zur Creative Commons Lizenz beifügen und angeben, ob Änderungen vorgenommen wurden.

Die in diesem Artikel enthaltenen Bilder und sonstiges Drittmaterial unterliegen ebenfalls der genannten Creative Commons Lizenz, sofern sich aus der Abbildungslegende nichts anderes ergibt. Sofern das betreffende Material nicht unter der genannten Creative Commons Lizenz steht und die betreffende Handlung nicht nach gesetzlichen Vorschriften erlaubt ist, ist für die oben aufgeführten Weiterverwendungen des Materials die Einwilligung des jeweiligen Rechteinhabers einzuholen.

Weitere Details zur Lizenz entnehmen Sie bitte der Lizenzinformation auf http://creativecommons.org/ licenses/by/4.0/deed.de.

\section{Literatur}

Abdul-Hussain, S. (2012). Genderkompetenz in Supervision und Coaching. Wiesbaden: VS.

v. Ameln, F. (2015). Interne Beratung - Gegenwart und Zukunft. Ein Plädoyer für interne Beratung als Schlüsselelement der Wandlungsfähigkeit von Organisationen. Gruppendynamik und Organisationsberatung, 46(1), 5-21.

v. Ameln, F. (2018). Führung und Beratung. Göttingen: Vandenhoeck \& Ruprecht.

Barrick, M.R., \& Mount, M. K. (1991). The big five personality dimensions and job performance: a metaanalysis. Personnel Psychology, 44(1), 1-26.

Bell, M.P., Özbilgin, M.F., Beauregard, T. A., \& Sürgevil, O. (2011). Voice, silence, and diversity in 21st century organizations: Strategies for inclusion of gay, lesbian, bisexual, and transgender employees. Human Resource Management, 50(1), 131-146.

Busse, S., \& Tietel, E. (2018). Mit dem Dritten sieht man besser. Göttingen: Vandenhoeck \& Ruprecht.

Ellwart, T., Russell, Y., \& Blanke, K. (2016). Führung als Doppelspitze: Co-Leitung erfolgreich managen. In J. Felfe \& R. van Dick (Hrsg.), Handbuch Mitarbeiterführung: Wirtschaftspsychologisches Praxiswissen für Fach- und Führungskräfte (S. 1-12). Berlin, Heidelberg: Springer.

Endres, S., \& Weibler, J. (2019). Plural Leadership: Eine zukunftsweisende Alternative zur One-ManShow. Berlin, Heidelberg: Springer.

Fildhaut, B. (2013). Herausforderung Doppelspitze: Privates und Berufliches mischen sich. Organisationsberatung, Supervision, Coaching, 20(3), 261-271.

Fröse, M. W., \& Bauer, A. (2019). Ein kassandrischer Zwischenruf - ausgewählte Phänomene von Führung in Expertenorganisationen am Beispiel von Hochschulen. In P. Kels \& S. Kaudela-Baum (Hrsg.), Experten führen: Modelle, Ideen und Praktiken für die Organisations- und Führungsentwicklung (S. 127-151). Wiesbaden: Springer.

Fröse, M. W., \& Szebel-Habig, A. (2009). Mixed Leadership: mit Frauen in die Führung! Bern: Haupt.

Giernalczyk, T., \& Möller, H. (2018). Entwicklungsraum: Psychodynamische Beratung in Organisationen. Göttingen: Vandenhoeck \& Ruprecht.

Gronn, P. (2002). Distributed leadership as a unit of analysis. The Leadership Quarterly, 13(4), $423-451$.

Guzzo, R.A., \& Dickson, M.W. (1996). Teams in organizations: Recent research on performance and effectiveness. Annual Review of Psychology, 47(1), 307-338.

de Haan, E., Grant, A.M., Burger, Y., \& Eriksson, P.O. (2016). A large-scale study of executive and workplace coaching: the relative contributions of relationship, personality match, and self-efficacy. Consulting Psychology Journal: Practice and Research, 68(3), 189. 
Hartmann, J., Boerner, S., \& Hüttermann, H. (2013). Mehr Chefinnen, mehr Erfolg? Personalmagazin, 15(4), 24-27.

Hegewald, J. (2017). Nimm 2 - Moderne Führung und Agilität. https://www.agil-gefuehrt.de/nimm-2/. Zugegriffen: 15. Okt. 2020.

Himmen, E. (2019). Topsharing. Wiesbaden: Springer.

Hinz, O. (2014). Das Führungsteam: wie wirksame Kooperation an der Spitze gelingt. Wiesbaden: Springer Gabler.

Hockling, S. (2019). Echte Diversität als Geschäftsgrundlage. In D. Brommer, S. Hockling \& A. Leopold (Hrsg.), Faszination New Work: 50 Impulse für die neue Arbeitswelt (S. 57-64). Wiesbaden: Springer.

Hossiep, R., \& Paschen, M. (2003). Das Bochumer Inventar zur berufsbezogenen Persönlichkeitsbeschreibung: BIP. Göttingen: Hogrefe.

Joshi, A., \& Roh, H. (2009). The role of context in work team diversity research: a meta-analytic review. Academy of Management Journal, 52(3), 599-627.

Karlshaus, A. (2016). Führung in Teilzeit: Herausforderung und Chance für eine nachhaltige Personalpolitik. In T. Doyé (Hrsg.), CSR und Human Resource Management (S. 69-97). Berlin, Heidelberg: Springer Gabler.

Kearney, E., \& Voelpel, S. C. (2012). Diversity research—what do we currently know about how to manage diverse organizational units? In B. Beham, J. Schwalbach \& C. Straub (Hrsg.), Managing diversity in organizations (S. 3-18). Wiesbaden: Springer Gabler.

van Knippenberg, D., \& Schippers, M. C. (2007). Work group diversity. Annual Review of Psychology, 58, 515-541.

van Knippenberg, D., de Dreu, C. K. W., \& Homan, A.C. (2004). Work group diversity and group performance: an integrative model and research agenda. Journal of Applied Psychology, 89(6), 1008.

Kotte, S., Bick, E., Benecke, C., \& Möller, H. (2019). Operationalisierte Psychodynamische Diagnostik im Arbeitskontext. Organisationsberatung, Supervision, Coaching, 26(1), 67-83.

Kuark, J. K. (2003). Gemeinsam an der Spitze - Das Modell TopSharing. Lenzburg: JKK Consulting.

Laloux, F. (2015). Reinventing organizations: ein Leitfaden zur Gestaltung sinnstiftender Formen der Zusammenarbeit. München: Vahlen.

Mayring, P. (2010). Qualitative Inhaltsanalyse. In G. May \& K. Mruck (Hrsg.), Handbuch Qualitative Forschung in der Psychologie (S. 601-613). Wiesbaden: VS.

Möller, H. (2012). Vertrauens- und Misstrauenskulturen in Organisationen. In H. Möller (Hrsg.), Vertrauen in Organisationen: Riskante Vorleistung oder hoffnungsvolle Erwartung? (S. 13-27). Wiesbaden: Springer VS.

Nagel, C. (2012). Behavioral Strategy und die Methode der Psychodynamischen Strategieentwicklung. In I. Bamberger \& T. Wrona (Hrsg.), Strategische Unternehmensberatung: Konzeptionen - Prozesse Methoden (6. Aufl. S. 243-261). Wiesbaden: Springer Gabler.

Nagel, C. (2013). Behavioral strategy: Denken und Fühlen im Entscheidungsprozess; das Unbewusste und der Unternehmenserfolg. Bonn: Unternehmer Medien.

Nezik, A. (2020). Schon wieder weg. DIE ZEIT, Nr. 19 (S. 20).

Pearce, C.L., \& Sims Jr, H.P. (2002). Vertical versus shared leadership as predictors of the effectiveness of change management teams: an examination of aversive, directive, transactional, transformational, and empowering leader behaviors. Group Dynamics: Theory, Research, and Practice, 6(2), 172.

Rastetter, D., \& Jüngling, C. (2018). Frauen, Männer, Mikropolitik. Göttingen: Vandenhoeck \& Ruprecht.

Reid, W., \& Karambayya, R. (2009). Impact of dual executive leadership dynamics in creative organizations. Human Relations, 62(7), 1073-1112.

Schippers, M. (2012). Why team reflexivity works. RSM Discovery-Management Knowledge, 12(4), 18-19.

Schippers, M.C., West, M.A., \& Dawson, J.F. (2015). Team reflexivity and innovation: the moderating role of team context. Journal of Management, 41(3), 769-788.

Schreyögg, A. (2005a). Coaching von Doppelspitzen: Anleitung für den Coach. Frankfurt/M.: Campus.

Schreyögg, A. (2005b). Coaching von Doppelspitzen - Wann sind Formen von Mediation zu integrieren? Organisationsberatung, Supervision, Coaching, 12(3), 217-230.

Schreyögg, A. (2009). Die Bedeutung der Familienkonstellationen im Coaching. In C. J. Schmidt-Lellek \& A. Schreyögg (Hrsg.), Praxeologie des Coaching (S. 76-87). Wiesbaden: VS.

Schreyögg, A. (2013). Übertragung und Gegenübertragung im Coaching. Organisationsberatung, Supervision, Coaching, 20(4), 409-423.

Stevens, M.J., \& Campion, M. A. (1994). The knowledge, skill, and ability requirements for teamwork: Implications for human resource management. Journal of Management, 20(2), 503-530. 
Stevens, M. J., \& Campion, M. A. (1999). Staffing work teams: Development and validation of a selection test for teamwork settings. Journal of Management, 25(2), 207-228.

Thatcher, S. M.B., \& Patel, P.C. (2012). Group faultlines: A review, integration, and guide to future research. Journal of Management, 38(4), 969-1009.

Vine, B., Holmes, J., Marra, M., Pfeifer, D., \& Jackson, B. (2008). Exploring co-leadership talk through interactional sociolinguistics. Leadership, 4(3), 339-360.

Volk, T. (2019). Spielen, um zu gewinnen. Göttingen: Vandenhoeck \& Ruprecht.

Weber, V., Sadwornych, K., Stoye-Mingers, G., \& Jarosch, A. A. (2016). Geheimtipps für Chefs: Was Business-Frauen wirklich denken. In P. Buchenau (Hrsg.), Chefsache Männer. Frauen machen Männer erfolgreich (S. 281-307). Wiesbaden: Springer Gabler.

Wimmer, R. (2004). Organisation und Beratung: systemtheoretische Perspektiven für die Praxis. Heidelberg: Carl-Auer.

Worchel, S., Rothgerber, H., Day, E. A., Hart, D., \& Butemeyer, J. (1998). Social identity and individual productivity within groups. British Journal of Social Psychology, 37(4), 389-413.

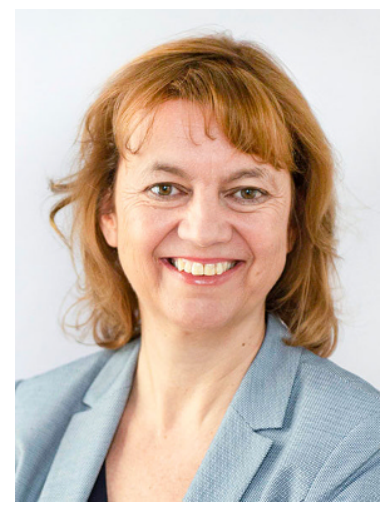

Christiane Münderlein Erste Vorständin des Evangelischen KITAVerbands Bayern mit Schwerpunkt Bildungs- und Sozialpolitik, verantwortlich für Organisations- und Fachberatung sowie Fort- und Weiterbildung von 800 Trägern mit 15.000 Mitarbeitenden. Nebenberuflich tätig als Coach von Führungskräften und Vorstandsteams sowie als Supervisorin von Berater/innen. Studium des Sozialwesens an der Evangelischen Hochschule Nürnberg und Coaching, Organisationsberatung, Supervision an der Universität Kassel. www.christiane-muenderlein.de, www.evkita-bayern.de. 